Results: The main issues raised by the respondents included the following:

- The interpretation of definitions introduced in the new model for the mass casualty preparedness model and the terrorist attack approach differed among respondents.

- All respondents supported the six points of departure in the CRBN and terrorist attack approach.

- Awareness of optimal personal safety ('safety first principle') specific for CBRN and terrorism is lacking.

- Respondents reported that several rescue workers did not feel competent to perform specific newly introduced tasks, such as the command and control of the first ambulance arriving at the scene and the coordination task of emergency transport by the dispatch nurse.

- Current regional differences in preparedness may complicate interregional collaboration.

Discussion: As the approach is new and experience is primarily based on the outcome of exercises, the systematic planning and evaluation of exercises, and sharing of opinions and knowledge, as a result, is important to ensure an unambiguous approach in a real situation.

Prehosp. Disaster Med. 2019;34(Suppl. 1):s79-s80

doi:10.1017/S1049023X19001675

\section{Relating the All Hazard Approach of Hospitals to that of Public Authorities}

Dr. Pieter van der Torn ${ }^{2}, M r$. Roel Geene ${ }^{1}$, Dr. Dennis den Hartog $^{3}$

1. Trauma Centre Southwest, Erasmus MC, University Medical Center Rotterdam, Rotterdam, Netherlands, Rotterdam, Netherlands

2. Itineris Consultancy, Rotterdam, The Netherlands

3. Trauma Research Unit, Department of Surgery, Erasmus MC, University Medical Center Rotterdam, Rotterdam, The Netherlands

Introduction: Hospitals and the healthcare sector suffer from chronic work overload and personnel shortages in many nations. This poses strong incentives to rationalize all activities not directly related to care, such as the preparations for disasters and other hazards. One such rationalization is to turn from a rulebased to a risk-based approach. However, the risk landscape of hospitals and the relationship to the risk landscape of public authorities are ill-defined. Health Care Coalitions (HCCs) are in a good position to fill this gap and serve as an intermediary. We developed a scheme for defining the risk landscape of HCCs and its members and performed a prioritization process. Aim: Objectives were to develop a knowledge platform of hospitals on risk assessment, promote integrated risk management by the HCC and its members, and determine the limiting (response) state for all relevant hazards.

Methods: We put maximum effort in limiting the time consumption for hospitals and align with the regular practices in hospitals for business continuity management. Strong points included the cooperation with the public authorities for safety and for health, a stepwise development of risk awareness and stepwise guidance for the assessment by hospitals, and formalization of the scenario-selection and choice of priorities by the HCC board.

Results: A gross list of $(>230)$ safety hazards was produced along with a netlist of $(>80)$ hazards relevant to health care.
In addition, an impact-scale for the continuity of care serving as a measurement stick for all health care sectors was developed. Risk diagrams were developed to present the results in a simple and clear format.

Discussion: The HCC risk landscape served its purpose in improving the mutual understanding with the public authorities. The formal assessment provides a solid basis for operational planning, education, training, and future investments.

Prehosp. Disaster Med. 2019;34(Suppl. 1):s80

doi:10.1017/S1049023X19001687

\section{Safer Hospitals in North Queensland - Assessment of Resilience \\ Mrs. Jenny Luke ${ }^{1,2}$, Assoc. Professor Richard Franklin ${ }^{2}$, Assoc. Professor Peter Aitkin ${ }^{3}$, Ms. Joanne Dyson ${ }^{3}$ \\ 1. Townsville Hospital and Health Service, Douglas, Australia \\ 2. James Cook University, Anton Breinl Research Centre for Health Systems Strengthening, Townsville, Australia \\ 3. Health Health Disaster Management Unit - Department of Health, Brisbane, Australia}

Introduction: Hospitals are fundamental infrastructure, and when well-designed can provide a trusted place of refuge and a central point for health and wellbeing services in the aftermath of disasters. The ability of hospitals to continue functioning is dependent on location, the resilience of buildings, critical systems, equipment, supplies, and resources as well as people. Working towards ensuring that the local hospital is resilient is essential in any disaster management system and the level of hospital resilience can be used as an indicator in measuring community resilience. The most popular measure of hospital resilience is the World Health Organisation's Hospital Safety Index (HSI) used in over 100 countries to assess and guide improvements to achieve structurally and functionally disaster resilient hospitals. Its purpose is to promote safe hospitals where services "remain accessible and functioning at maximum capacity, and with the same infrastructure, before, during and immediately after the impact of emergencies and disasters." It identifies likely high impact hazards, vulnerabilities, and mitigation/improvement actions.

Aim: The HSI can be a valuable tool as part of the 2015-2030 Sendai Framework for Disaster Risk Reduction. However, to date, it has been used infrequently in developed countries. This project pilots the application of the HSI across seven facilities in a North Queensland health service (an area prone to cyclones and flooding), centered on a tertiary referral center, each providing 24-hour emergency health services.

Results: Key indicators of resilience and the result of the audit will be discussed within geographical and cultural contexts, including the benefits of the HSI in augmenting existing hospital assessment and accreditation processes to identify vulnerabilities and mitigation strategies.

Discussion: The research outcomes are to be used by the health service to improve infrastructure and provide anticipated community benefits, especially through the continuation of health services post disasters.

Prehosp. Disaster Med. 2019;34(Suppl. 1):s80

doi:10.1017/S1049023X19001699 